BMJ Open Ophthalmology

\section{Exploring the precision of femtosecond laser-assisted descemetorhexis in Descemet membrane endothelial keratoplasty}

To cite: Pilger D, von Sonnleithner C, Bertelmann E, et al. Exploring the precision of femtosecond laser-assisted descemetorhexis in Descemet membrane endothelial keratoplasty. BMJ Open Ophthalmology 2018;3:e000148. doi:10.1136/ bmjophth-2018-000148

DP and CvS contributed equally.

Received 17 January 2018 Revised 5 0ctober 2018 Accepted 19 November 2018
Check for updates

\section{(c) Author(s) (or their} employer(s)) 2018. Re-use permitted under CC BY-NC. No commercial re-use. See rights and permissions. Published by BMJ.

Department of Ophthalmology, Charité-Medical University Berlin, Berlin, Germany

\section{Correspondence to} Dr Daniel Pilger; daniel.pilger@ charite.de

\section{ABSTRACT \\ Objective Descemet membrane endothelial keratoplasty} (DMEK) remains a challenging technique. We compare the precision of femtosecond laser-assisted DMEK to manual DMEK.

Methods and Analysis A manual descemetorhexis (DR) of $8 \mathrm{~mm}$ diameter was compared with a femtosecond laser-assisted DR of the same diameter (femto-DR) in 22 pseudophakic patients requiring DMEK. We used OCT images with a centred xy-diagram to measure the postoperative precision of the DR and the amount of endothelial denuded area. Endothelial cell loss (ECL) and best corrected visual acuity were measured 3 months after surgery.

Results In the manual group, the median error of the DR was $7 \%$ (range $3 \%-16 \%$ ) in the $x$-diameter and $8 \%$ (range $2 \%-17 \%$ ) in the $y$-diameter. In the femto group, the median error in the respective $x$ and $y$-diameters was $1 \%$ (range $0.4 \%-3 \%$ ) and $1 \%$ (range $0.006 \%-2.5 \%$ ), smaller than in the manual group $(p=0.001)$. Endothelial denuded areas were larger in the manual group $\left(11.6 \mathrm{~mm}^{2}\right.$, range $\left.7.6-18 \mathrm{~mm}^{2}\right)$ than in the femto group $\left(2.5 \mathrm{~mm}^{2}\right.$, range $1.2-$ $\left.5.9 \mathrm{~mm}^{2}\right)(\mathrm{p}<0.001)$. The ECL was $21 \%$ (range $5 \%-78 \%$ ) in the manual DR and $17 \%$ (range $6 \%-38 \%$ ) in the femto-DR group $(p=0.351)$. The median visual acuity increased from $0.4 \log$ MAR (range 0.6-0.4 logMAR) in both groups to 0.1 logMAR (range 0.4-0 logMAR) in the manual group and to 0.1 logMAR (range $0.3-0$ logMAR) in the femto group $(p=0.461)$. Three rebubblings were required in the manual group, whereas the femto group required only one.

Conclusion The higher precision of the femto-DR bears the potential to improve DMEK surgery.

\section{INTRODUCTION}

An important step in Descemet membrane endothelial keratoplasty (DMEK) surgery is the incision of the host's Descemet membrane (DM), the descemetorhexis (DR). ${ }^{1}$ The surgeon accesses the anterior chamber and manually cuts through host DM in a circular motion. The incision marks the area where the host's DM will be removed and where the graft will be attached. Whereas creating a DR that precisely fits the size of the transplant is ideal, it bears the risk of creating

\section{Significance of the study}

What is already known about this subject?

Femtosecond laser-assisted descemetorhexis (DR) is a safe method for facilitating Descemet membrane endothelial keratoplasty (DMEK) surgery.

\section{What are the new findings?}

Femto-DR parameters have to ensure a complete incision of the Descemet membrane to avoid radial tears. The precision of the femto-DR exceeds manual $\mathrm{DR}$, which could help decrease endothelial denuded areas.

\section{How might these results change the focus of research or clinical practice?}

Femtosecond laser-assisted DR could be used by surgeons wishing to increase the precision of DMEK surgery and exploring ways of improving postoperative outcomes.

areas with endothelial overlap. Such areas are a known risk factor for graft detachment. ${ }^{23}$ The general recommendation is to create a DR that exceeds the size of the transplant. ${ }^{3}$

We have previously described a novel technique for facilitating DMEK surgery that uses a femtosecond laser to perform the DR. ${ }^{4}$ This method has shown a similar efficacy to the standard DMEK technique with the potential of reducing flap detachments. ${ }^{5}$ In the current study, we compare femtosecond laser-assisted DMEK surgery with standard DMEK surgery in order to explore its potential to increase the precision of the procedure.

\section{METHODS}

Eleven pseudophakic patients with Fuchs endothelial dystrophy and no history of intraocular surgery (excluding cataract surgery) received DMEK surgery with femto-DR (femto group). We compared them with 11 patients receiving a manual DR (manual group) and followed them up for 3 months. 
All surgical procedures were performed by the same surgeon (NT) and remained the same in both groups except for the DR. We performed the femto-DR as described earlier ${ }^{4}$ using the LenSx system (Alcon Laboratories, Fort Worth, Texas, USA). Proprietary energy and spot separation parameters were set to the standard settings of the manufacturer as used in cataract surgery (energy $5 \mathrm{~mJ}$, tang spot separation $4 \mu \mathrm{m}$ and layer separation $3 \mu \mathrm{m}$ ). For all patients in the femto group, the depth of the cylindrical pattern was set to $100 \mu \mathrm{m}$ into the anterior chamber. The depth of the cylindrical pattern into the corneal stroma was allowed to vary; in six patients, the depth was set to $100 \mu \mathrm{m}$. In the remaining patients, the depth was reduced to $75 \mu \mathrm{m}(\mathrm{n}=3)$ and $60 \mu \mathrm{m}(\mathrm{n}=2)$. The $\mathrm{DR}$ diameter was set to $8.0 \mathrm{~mm}$ - the maximum diameter allowed by the system. In the manual group, we used a corneal maker (Storz Ophthalmics) to mark a circle of $8.0 \mathrm{~mm}$ in diameter on the patients' cornea. Subsequently, we manually performed the DR along the circle using an Althaus/Cartsberg scraper with irrigation system (Geuder, Heidelberg, Germany). After either type of DR, clear corneal incision without sutures was used to access the anterior chamber. The DM was manually peeled off. Cultured grafts with a minimum central endothelial cell (EC) density of 2000 cells $/ \mathrm{mm}^{2}$ were provided by the Cornea Bank Berlin. As described by Melles $e t a l,{ }^{1}$ we stripped the donor DM approximately 1 hour before DMEK surgery, and cut it to a diameter of $8.0 \mathrm{~mm}$ using a trephine. The graft lamella was injected into the anterior chamber using a glass injector. After unfolding, the anterior chamber was filled with air which we partially removed approximately 2 hours after completion of the surgery.

The quality of the DR and graft detachments was assessed up to 3 months after surgery, and in clear corneas without signs of oedema, using the Spectralis optical coherence tomography (OCT) device with an anterior chamber attachment with a resolution of 11 $\mu \mathrm{m}$ per pixel (Spectralis OCT, Heidelberg engineering Family acquisition Module 5.3.3.0, Heidelberg engineering Viewing Module 5.3.2.0 and Heidelberg Eye explorer V.1.6.4.0 software; Heidelberg Engineering, Heidelberg, Germany). We estimated DR parameters using enface OCT images by centring two perpendicular lines on the cornea to measure the horizontal (x-diameter) and vertical (y-diameter) extension of the DR. We used the difference between the measured diameters and the preset $8.0 \mathrm{~mm}$ diameter to estimate the error of the DR. To estimate endothelial denuded areas and area of endothelial overlap, we used the area measurement tool of the OCT. Measurements were performed by two independent researchers and we used the mean of both measurements. EC density was measured 3 months after surgery using the specular microscope, Nidek CEM-530 (NIDEK, Japan) and it was compared with the EC density of the transplant prior to surgery in order to calculate endothelial cell loss (ECL). We used the median and range to describe our findings, and the Mann-Whitney U test for comparisons.

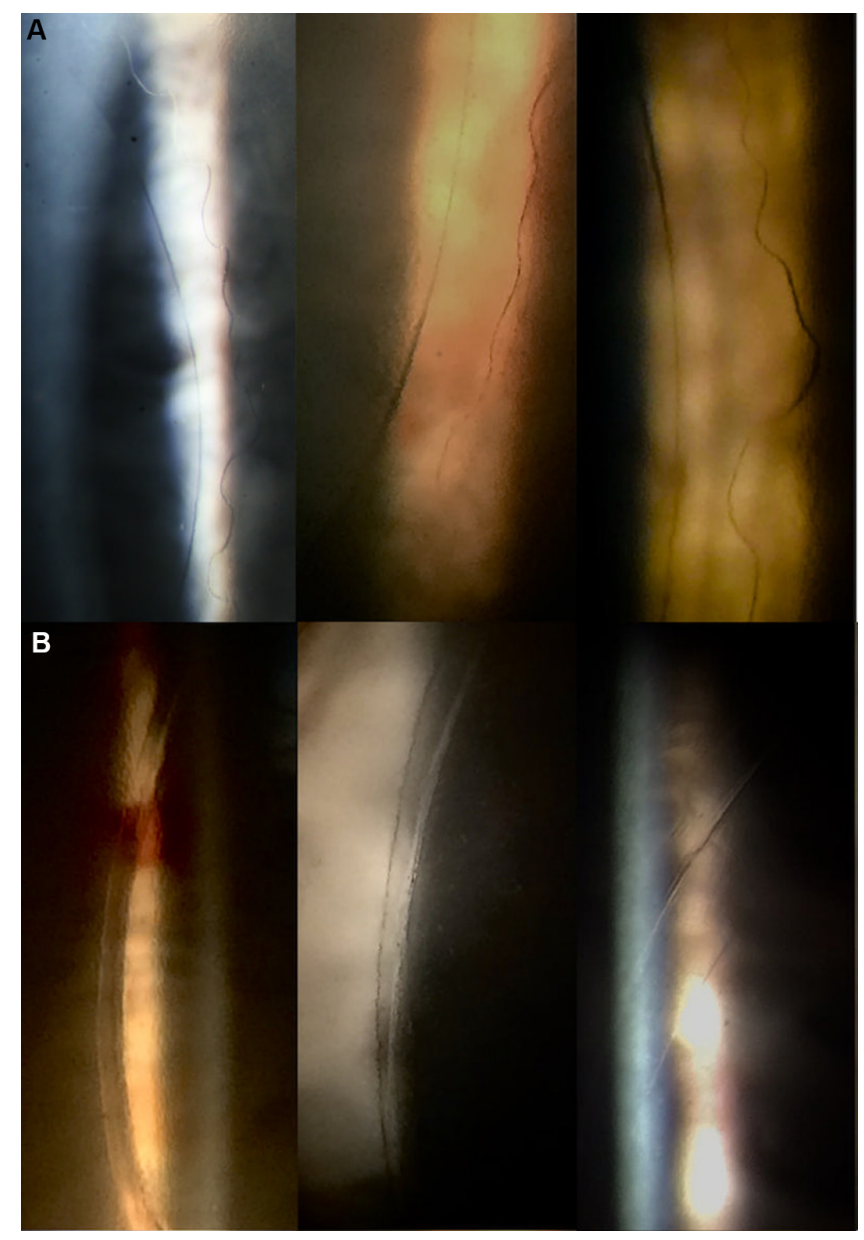

Figure 1 Slit lamp examination of $(A)$ manual descemetorhexis and (B) femto descemetorhexis.

\section{RESULTS}

\section{DR creation}

Slit lamp and OCT examination showed a scalloped pattern for manual DR (figures 1a and 2a). In the femto group, the edges of the DR were clear-cut and parallel to the graft (figures $1 b$ and $2 b$ ). OCT images revealed a complete cut edge in five patients with a femto-DR depth of $100 \mu \mathrm{m}$. In the remaining patients, OCT images showed incomplete cut edges (figure 3). In these patients, the femto-DR left DM bridges in the temporal $(n=4)$ and

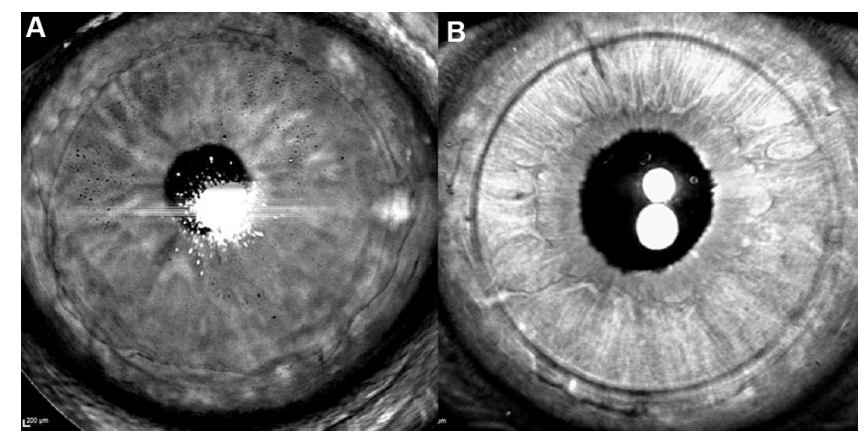

Figure 2 Enface optical coherence tomography (OCT) images of $(A)$ manual descemetorhexis and $(B)$ femto descemetorhexis. 


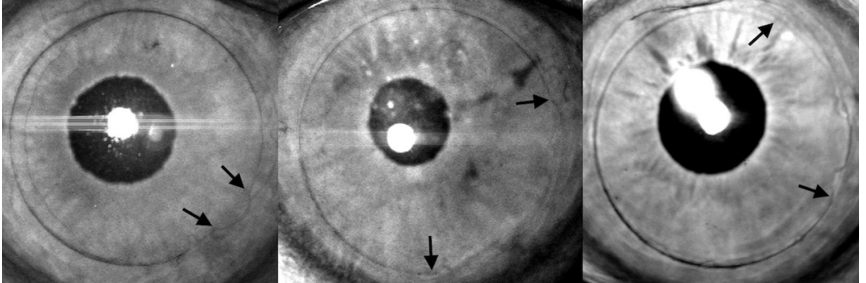

Figure 3 Enface optical coherence tomography (OCT) images of incomplete femto descemetorhexis. Arrows show the extension of radial tears.

nasal $(n=2)$ parts of the hosts' cornea that resulted in small radial tears when the DM was removed (figure 3). No edge lifts or stromal scars were visible on OCT images of the femto-DR.

The manual DR showed large variations in the achieved diameter with a median error in the $\mathrm{x}$ and $\mathrm{y}$-diameters of $7 \%$ (range $3 \%-16 \%$ ) and $8 \%$ (range $2 \%-17 \%$ ), respectively (table 1 ). In the femto group, the DR closely approximated to the intended $8.0 \mathrm{~mm}$ diameter with a median error of $1 \%$ (range $0.4 \%-3 \%$ ) in the x-diameter and $1 \%$ (range $0.006 \%-2.5 \%$ ) in the y-diameter (table 1 ). As a result, the maximum distance between the rhexis and the edge of the transplant was smaller in the femto group (table 1) and hence denuded parts were smaller in the femto group than in the manual group (table 1). Endothelial overlap occurred only twice in the manual group with a maximum area of overlap of $15 \mathrm{~mm}^{2}$. In the femto group, it occurred in all observations, but with only $5.9 \mathrm{~mm}^{2}$ the largest area of overlap was considerably smaller than in the manual group (table 1).

\section{Postoperative slit lamp examination and visual acuity}

In all patients, surgery resulted in clear corneas 1 month after surgery. No corneal abrasions were observed either after the laser procedure or during the postoperative follow-up. All patients had normal pupil examination, with no evidence of iris damage, atrophy or transillumination defects.

The median BCVA increased from 0.4 logMAR (range 0.6-0.4 $\log$ MAR) in both groups to $0.1 \log$ MAR (range 0.4-0 $\log$ MAR) in the manual group and to $0.1 \log$ MAR (range 0.3-0 logMAR) in the femto group.

\section{Complications and adverse events}

In the femto group, six patients showed incomplete DR. No further intraoperative complications occurred in either group. In the manual group, three patients required a rebubbling due to a flap detachment larger than two clock hours; two occurred in patients with endothelial overlap. In the femto group, only one graft detachment was noted. This did not occur in an area of endothelial overlap. The ECL was slightly higher in the manual group $(21 \%$, range $5 \%-78 \%)$ than in the femto group $(17 \%$, range $6 \%-38 \%)(\mathrm{p}=0.351)$.

\section{DISCUSSION}

In the absence of radial tears, femto-DR can produce a precise rhexis of stable diameter. ${ }^{4}$ Similar to femtosecond laser-assisted capsulotomy in cataract surgery, ${ }^{6}$ the femto-DR exceeds the precision of the manual DR (in patients without radial tears). In addition to the precise diameter, femto-DR also produces edges that are clear-cut whereas the edges produced by manual DR are scalloped. Such clear-cut edges in combination with the less variable diameter leads to a reduction in endothelial denuded areas. As corneal ECs migrate to denuded areas, ${ }^{78}$ smaller denuded areas could increase central endothelial density. In contrast, endothelial overlap was more frequently observed in the femto group due to the smaller diameter of the DR. Overlapping areas, however, were relatively small compared with the overlapping areas seen in the manual group and it is possible that such small endothelial overlap does not cause graft detachment over more than one clock hour. To avoid endothelial overlap, the LenSx laser system could be modified allowing DR diameters exceeding the diameter of the transplant. ${ }^{3}$ Different models of femtosecond lasers such as the Wavelight FS200 or the IntraLase FS150 have already implemented a wider diameter for the DR in their software. The diameter of the DR could be chosen to fit the surgeon's ability to centre the graft within the DR. This would reduce areas of overlap and could still result in less endothelial denuded area than manual DR, due to the small variation in diameter and the evenly curved edges of the femto-DR.

All femtosecond lasers use a patient interface that docks to the patient's cornea and causes some distortion in the anterior and posterior corneal surface. In addition, the corneal thickness is not uniform at a given radius from the geometric centre of the cut. In our study, incomplete DR occurred mostly when the depth of the cylindrical cut into the corneal stroma was set below $100 \mu \mathrm{m}$. Using an

Table 1 Descemetorhexis parameters

\begin{tabular}{llcc}
\hline Median $($ range) & Manual & Femto & P value \\
\hline X-diameter of rhexis $(\mu \mathrm{m})$ & $8600(8257-9343)$ & $8082(7924-8302)$ & 0.001 \\
\hline Y-diameter of rhexis $(\mu \mathrm{m})$ & $8700(8159-9424)$ & $8094(7995-8114)$ & 0.001 \\
$\begin{array}{l}\text { Maximum distance between rhexis and transplant } \\
(\mu \mathrm{m})\end{array}$ & $627(128-1209)$ & $330(166-737)$ & 0.087 \\
Median area of denuded parts $\left(\mathrm{mm}^{2}\right)$ & $11.6(7.6-18)$ & $2.5(1.2-5.9)$ & $<0.001$ \\
Median area of endothelial overlap $\left(\mathrm{mm}^{2}\right)$ & $0(0-15)$ & $2.5(1.3-5.9)$ & 0.225 \\
\hline
\end{tabular}


IntraLase laser with a cylindrical cut of $100 \mu \mathrm{m}$ depth into the corneal stroma, Einan-Lifshitz et al did not report any radial tears when employing femto-DR. ${ }^{5}$ This suggests that a depth of below $100 \mu \mathrm{m}$ may not compensate for the distortion of the posterior corneal surface and the variation in corneal thickness. While a small margin reduces the impact on the posterior corneal stroma, this can result in an incomplete DR which in turn compromises the precision of the DR. Ideally, the cylindrical depth should allow a complete incision of the DM but also minimise the incision depth into the corneal stroma.

This exploratory study does not intend to prove superiority of the femto-DR. The limited sample size restricts the generalisation of our findings. This is particularly true for rebubbling rates and visual acuity, as these could be strongly affected by the variation in cylindrical pattern that was employed in this study. The same is true for ECL, which is known to be influenced by many confounders that were not considered in this study. In addition, we did not measure the actual size of the transplant but assumed that trephination would lead to a graft with $8.0 \mathrm{~mm}$ in diameter. Variation in transplant diameter could have biased our measurements. Also, measuring areas on a two-dimensional enface OCT image meant that we are not taking the curved nature of the cornea into account. This could have led to an underestimation of our measurements. Nevertheless, measurement bias would have occurred in both groups, therefore affecting the absolute values of our measurements, but not the tendency seen in the comparison.

In conclusion, femto-DR settings have to ensure a complete incision of the DM. When radial tears are avoided, femto-DR might reduce endothelial denuded areas and could improve DMEK surgery.

Acknowledgements DP is a participant in the BIH-Charité Clinician Scientist Program funded by the Charité-Universitätsmedizin Berlin and the Berlin Institute of Health.
Contributors DP and CvS conducted the study, performed the analysis and wrote the first draft of the manuscript. EB, AKBM and AMJ contributed to the analysis and interpretation of the findings and added to the writing of the manuscript. NT conceived the study, performed the surgeries, interpreted the findings and contributed to the writing of the final manuscript. DP, CvS and NT act as guarantors for the overall content of this work.

Funding Consumables for the LenSx femtosecond laser were provided free of charge by Alcon Laboratories (Fort Worth, Texas, USA).

Competing interests DP and NT received financial support from Alcon Laboratories for presentations at meetings.

Patient consent for publication Not required.

Ethics approval Charite Universitätsmedizin Ethikkommission.

Provenance and peer review Not commissioned; externally peer reviewed.

Open access This is an open access article distributed in accordance with the Creative Commons Attribution Non Commercial (CC BY-NC 4.0) license, which permits others to distribute, remix, adapt, build upon this work non-commercially, and license their derivative works on different terms, provided the original work is properly cited, appropriate credit is given, any changes made indicated, and the use is non-commercial. See: http://creativecommons.org/licenses/by-nc/4.0

\section{REFERENCES}

1. Melles GR, Ong TS, Ververs B, et al. Descemet membrane endothelial keratoplasty (DMEK). Cornea 2006;25:987-90.

2. Brockmann T, Brockmann C, Maier AK, et al. Clinicopathology of graft detachment after Descemet's membrane endothelial keratoplasty. Acta Ophthalmol 2014;92:e556-e561.

3. Tourtas T, Schlomberg J, Wessel JM, et al. Graft adhesion in descemet membrane endothelial keratoplasty dependent on size of removal of host's descemet membrane. JAMA Ophthalmol 2014;132:155-61.

4. Pilger D, von Sonnleithner C, Bertelmann E, et al. Femtosecond laserassisted descemetorhexis: a novel technique in descemet membrane endothelial keratoplasty. Cornea 2016;35:1274-8.

5. Einan-Lifshitz A, Sorkin N, Boutin T, et al. Comparison of femtosecond laser-enabled descemetorhexis and manual descemetorhexis in descemet membrane endothelial keratoplasty. Cornea 2017;36:767-70.

6. Nagy Z, Takacs A, Filkorn T, et al. Initial clinical evaluation of an intraocular femtosecond laser in cataract surgery. J Refract Surg 2009;25:1053-60.

7. Hos D, Heindl LM, Bucher F, et al. Evidence of donor corneal endothelial cell migration from immune reactions occurring after descemet membrane endothelial keratoplasty. Cornea 2014;33:331-4.

8. Jacobi C, Zhivov A, Korbmacher J, et al. Evidence of endothelial cell migration after descemet membrane endothelial keratoplasty. Am J Ophthalmol 2011;152:537-42. 